Journal of

Business \&

Economic

Statistics

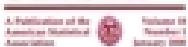

\section{Journal of Business \& Economic Statistics}

ISSN: 0735-0015 (Print) 1537-2707 (Online) Journal homepage: https://www.tandfonline.com/loi/ubes20

\title{
A Locally Optimal Seasonal Unit-Root Test
}

\section{Mehmet Caner}

To cite this article: Mehmet Caner (1998) A Locally Optimal Seasonal Unit-Root Test, Journal of Business \& Economic Statistics, 16:3, 349-356

To link to this article: https://doi.org/10.1080/07350015.1998.10524774

Published online: 02 Jul 2012.

Submit your article to this journal

山 Article views: 43

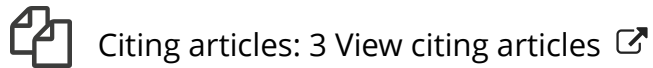




\title{
A Locally Optimal Seasonal Unit-Root Test
}

\author{
Mehmet CANER \\ Department of Economics, Bilkent University, Ankara, Turkey
}

\begin{abstract}
This article proposes a locally best invariant test of the null hypothesis of seasonal stationarity against the alternative of seasonal unit roots at all or individual seasonal frequencies. An asymptotic distribution theory is derived and the finite-sample properties of the test are examined in a Monte Carlo simulation. My test is also compared with the Canova and Hansen test. The proposed test is superior to the Canova and Hansen test in terms of both size and power.
\end{abstract}

KEY WORDS: Locally best invariant test; Maximum likelihood estimation; Monte Carlo; Seasonal differencing filter; Seasonal stationarity.

Seasonality in economic time series can be viewed as either deterministic or stochastic. Fitting dummy variables is the usual way of handling seasonality in a deterministic way. To differentiate among various patterns of seasonality, statistical tests are introduced in the literature.

Hylleberg, Engle, Granger, and Yoo (HEGY) (1990) developed tests of the null hypothesis of a unit root at one or more seasonal frequencies against the alternative of stationary seasonality. Their test is an extension of the unit-root test of Dickey and Fuller (1979) from the zero frequency to the seasonal frequencies. This test has low power in finite samples near unit roots, so it is difficult to reject the false unit-root hypothesis at a single or a set of seasonal frequencies (Canova and Hansen 1995).

In the tests that are developed by Canova and Hansen (CH) (1995), stationary seasonality forms the null hypothesis. The alternative hypothesis is nonstationarity due to seasonal unit roots. They generalized the unit-root test of Kwiatkowski, Phillips, Schmidt, and Shin (KPSS) (1992) from the zero frequency to the seasonal frequencies. Their test statistics are Lagrange multiplier (LM) tests that are modified to include serially correlated and heteroscedastic processes. Only least squares techniques are needed in their LM-type test, and autocorrelation is handled by using a nonparametric adjustment. Tam and Reinsel (1995) also contributed to this literature by developing tests for moving average (MA) seasonal unit roots. Their test is mainly the extension of the Saikkonen and Luukkonnen (1993) unitroot test to seasonal frequencies.

In this article, $\mathbb{I}$ propose a test procedure in which the null hypothesis of stationary seasonality is tested against the alternative of seasonal nonstationarity. I generalize the unit-root test of Leybourne and McCabe (1994) from zero frequency to the seasonal frequency. To test the null hypothesis, I propose a locally best invariant test that is derived from the framework of King and Hillier (1985). The test statistics depend on the residuals, which are calculated via maximum likelihood and then using least squares. The large-sample distribution under the null is the generalized von Mises distribution that does not depend on the nuisance parameters.

There are two major differences between my test and the $\mathrm{CH}$ test for seasonal stability. In my test, autocorrelation is taken into account in a parametric way, but the $\mathrm{CH}$ test uses a nonparametric correction. Second, my test statistic is consistent to the order $N$, whereas the $\mathrm{CH}$ test is of order $N / z$, where $z$ is the lag truncation parameter. The nonparametric adjustment of autocorrelation suggested by Canova and Hansen (1995) fails to give good finite-sample performance when a large autoregressive (AR) component is present in the data. This problem is due to the significant truncation errors in the finite samples.

The aim of this article is to overcome this problem by introducing a test statistic that accounts for autocorrelation parametrically. A Monte Carlo exercise has been conducted to examine and compare the finite-sample properties of the proposed test with those of the $\mathrm{CH}$ test. It is shown that the proposed test has better size and power properties than the $\mathrm{CH}$ test in an AR type of autocorrelation.

Section 1 introduces the regression model. Section 2 presents the structural and reduced form of the model, and a locally best invariant test statistic is also derived for testing unit roots at the seasonal frequencies. The rest of the section develops the asymptotic distribution for this test statistic. In Section 3, a Monte Carlo exercise is conducted, and the size and power properties of the proposed test are compared to those of the $\mathrm{CH}$ test. Section 4 concludes the article. Proofs of the theorems are discussed in the Appendix. A GAUSS program for calculating test statistics is available on request from me.

\section{THE MODEL}

A linear time series model with stationary seasonality is considered:

$$
\Phi(L) y_{t}=\mu+S_{t}+e_{t}, \quad t=1,2, \ldots, N .
$$

In the preceding equation, $\Phi(L)=1-\phi_{1} L-\phi_{2} L^{2}-\cdots-$ $\phi_{p} L^{p}$ is a $p$ th-order AR polynomial in the lag operator $L$ with roots outside the unit circle, $y_{t}$ is real valued, and $S_{t}$ is a real-valued deterministic seasonal process of period $s$, in which $s$ is a positive even integer and error term $e_{t}$ is distributed as iid $\left(0, \sigma_{e}^{2}\right)$. The number of observations is $N$. If there are $T$ years of data, $N=T s$.

(C) 1998 American Statistical Association Journal of Business \& Economic Statistics July 1998 , Vol. 16, No. 3 
In this article, because $\mathbb{I}$ am interested in unit roots at the seasonal frequencies, I require that $y_{t}$ not have a unit root at the zero frequency because it is not very difficult to transform a series with nonstationarity at the zero frequency to a stationary series; various applications of this test will be possible. The autocorrelation in the series is accounted for by including the lagged terms in $y_{t}$.

A trigonometric representation is presented for the deterministic seasonal pattern $S$ :

$$
S_{t}=\sum_{j=1}^{q} f_{j t}^{\prime} \gamma_{j},
$$

where $q=s / 2(s=4$ for quarterly data and $s=12$ for monthly data) and, for $j<q, f_{j t}^{\prime}=$ $[\cos ((j / q) \pi t), \sin ((j / q) \pi t)]$, when $j=q, f_{q t}=\cos (\pi t)$. Expressing the right side of (2) in a vector,

$$
S_{t}=f_{t}^{\prime} \gamma
$$

where

$$
\gamma=\left(\begin{array}{c}
\gamma_{1} \\
\vdots \\
\gamma_{q}
\end{array}\right), \quad f_{t}=\left(\begin{array}{c}
f_{1 t} \\
\vdots \\
f_{q t}
\end{array}\right) \text {. }
$$

These $\gamma$ and $f_{t}$ vectors have $(s-1)$ elements. Substituting (3) into (1), the regression equation is

$$
\Phi(L) y_{t}=\mu+f_{t}^{\prime} \gamma+e_{t}, \quad t=1,2, \ldots, N .
$$

This representation allows seasonality to be a cylical process. At the seasonal frequency $j \pi / q$, the cylical processes are elements of $f_{t}$. Moreover, $f_{t}$ is a zero-mean process whenever $N$ is a multiple of $s$. The coefficients $\gamma_{j}$ represent the effect of each cycle on the deterministic seasonal component $S_{t}$. This cylical formulation of seasonality is common in the time series literature (Hannan 1970, p. 174; Harvey 1989, p. 42).

\section{THE TEST FOR SEASONAL UNIT ROOTS}

\subsection{The Structural Model and the Reduced Form}

To test whether seasonal patterns are stable or not, I need to present a specific alternative hypothesis. One form of the alternative hypothesis is to allow a unit root in $\gamma_{t}$. This idea was suggested by Hannan (1970) and used by Canova and Hansen (1995) and Leybourne and McCabe (1994). The structural model is

$$
\Phi(L) y_{t}=\mu+f_{t}^{\prime} \gamma_{t}+e_{t}, \quad t=1,2, \ldots, N,
$$

and

$$
\gamma_{t}=\gamma_{t-1}+u_{t},
$$

$\gamma_{0}$ fixed. It is assumed that $u_{t}$ is iid mean 0 , independent of $e_{t}$ and $f_{t}$ and its covariance matrix are

$$
E u_{t} u_{t}^{\prime}=\sigma_{u}^{2} G
$$

where $G$ is an $(s-1) \times(s-1)$ matrix and $\sigma_{u}^{2}$ is a scalar. The unit roots at different seasonal frequencies are determined by $G$ matrix. It can be easily seen from (6) and (7) that, whenever $\sigma_{u}^{2} \neq 0$, then there will be seasonal unit roots.
The structural model (6)-(7) is second-order equivalent in moments to the reduced-form model:

$$
\Phi(L) S(L) y_{t}=\mu^{\prime}+\Theta(L) \zeta_{t},
$$

where $\zeta_{t}$ is distributed $\left(0, \sigma_{\zeta}^{2}\right), S(L)=\sum_{j=0}^{s-1} L^{j}$ is a seasonal filter, and $\mu^{\prime}=s \mu$.

This last term $\Theta(L)$ is an $\operatorname{MA}(s-1)$ polynomial. The derivation of (9) can be obtained from me on demand. For the importance of this representation, the reader can consult Leybourne and McCabe (1994). This reduced form is very similar to the model used by Tam and Reinsel (1995). Their model did not, however, result in a test that can differentiate between unit roots at various seasonal frequencies and the zero frequency.

\subsection{The Hypothesis Test}

I wish to test whether the seasonal patterns are stable or not. In other words I need to develop a hypothesis so that I can determine whether a given series has stationary seasonality or not. One such hypothesis is a stationary $\operatorname{AR}(p)$ process, against the alternative of nonstationary seasonality. This can be easily formulated in terms of the structural model (6)-(7) as $H_{0}: \rho=0$ against $H_{1}: \rho>0$, where $\rho=\sigma_{u}^{2} / \sigma_{e}^{2}$. I shall examine the local departures from the null hypothesis in the following section.

Another task that I face is developing a test statistic. From the structural model (6)-(7) and using the framework of King and Hillier (1985), the locally best invariant test statistic for $H_{0}: \rho=0$ is

$$
D=\hat{\sigma}_{e}^{-2} N^{-2} \sum_{t=1}^{N} \hat{F}_{t}^{\prime} G \hat{F}_{t},
$$

where $\hat{F}_{t}=\sum_{i=1}^{t} f_{i} \hat{e}_{i}, \hat{\sigma}_{e}^{2}=\hat{e}^{\prime} \hat{e} / N$ is a consistent estimator of $\sigma_{e}^{2}$, and $\hat{e}$ is an $N \times 1$ vector of residuals $\hat{e}_{t}$.

The residuals $\hat{e}_{t}$ are obtained via the following procedure: First find the maximum likelihood estimates of $(\phi)$ from the fitted model,

$$
y_{t}^{*}=\mu^{\prime}+\sum_{l=1}^{p} \phi_{l} y_{t-l}^{*}+\Theta(L) \zeta_{t},
$$

where $y_{t}^{*}=S(L) y_{t}$. Then construct the series

$$
\bar{y}_{t}=y_{t}-\sum_{l=1}^{p} \phi_{l}^{*} y_{t-l},
$$

where $\phi_{l}^{*}$ are the maximum likelihood estimates of $\phi_{l}$ obtained from (11). Then regress $\bar{y}_{t}$ on an intercept and seasonal dummies to obtain $\hat{e}_{t}$. Even though I do not assume the normality of $e_{t}$, this is necessary for the "optimality" of the tests.

As pointed out by Saikonnen and Luukonnen (1993) and Leybourne and McCabe (1994), one wants to estimate $\phi_{l}$ consistently both under the null and the alternative hypothesis in the reduced form, so I use maximum likelihood estimation rather than ordinary least squares. Even 
though maximum likelihood estimation might have drawbacks, they are found to have no effect on the finite-sample properties of my test in the simulations that I have conducted in the former version of the article (Ansley and Newbold 1980; Galbraith and Zinde-Walsh 1994). An alternative way of estimation is the instrumental-variable technique. But this approach did not give good results in this case.

An important point is the structure of $G$ matrix. Different specifications of the alternative hypotheses depend on the structure of $G$. When the alternative hypothesis is unit roots at all seasonal frequencies, then $G$ must be nonsingular and $\gamma_{t}$ must be time varying. If the alternative hypothesis is unit roots at specific seasonal frequencies, then $G$ must be block diagonal with nonzero elements in only selected blocks and a subset of $\gamma_{t}$ is time varying.

\subsection{The Asymptotic Distribution}

If the alternative hypothesis is seasonal nonstationarity, then I should have a joint unit-root test at all seasonal frequencies. It was first suggested by Nyblom (1989) in the likelihood context and later applied to econometric models by Hansen $(1990,1992)$ that, when $G / \sigma_{e}^{2}=\left(\Omega^{f}\right)^{-1}$, then the asymptotic distribution of the test statistic is easy to evaluate. In the preceding discussion, $\Omega^{f}$ is the long-run covariance matrix of $f_{i} e_{i}$ (see Canova and Hansen 1995).

Because $e_{t}$ is serially uncorrelated and homoscedastic, I can use the consistent estimator

$$
\hat{\Omega}^{f}=\hat{\sigma}_{e}^{2}\left[\begin{array}{ccccc}
1 & 0 & 0 & 0 & 0 \\
0 & \frac{1}{2} & 0 & 0 & 0 \\
0 & 0 & \frac{1}{2} & 0 & 0 \\
0 & 0 & 0 & \ddots & 0 \\
0 & 0 & 0 & 0 & \ddots
\end{array}\right] .
$$

To study the large-sample distribution of $D$, I use the following notation: $\stackrel{d}{\rightarrow}$ denotes convergence in distribution, $W_{m}$ denotes a vector standard Brownian bridge of dimension $m$, and $V M(m)$ is a random variable obtained by the following operation:

$$
V M(m)=\int_{0}^{1} W_{m}(r)^{\prime} W_{m}(r) d r
$$

As was suggested by Canova and Hansen (1995), $V M(m)$ will be referred to as the generalized von Mises distribution with $m$ df. Critical values were given in table 1 of Canova and Hansen (1995). The main theorem of this section is proved in the Appendix.

Theorem 1. In (1), if $\Phi(L)$ is a finite AR polynomial in the lag operator with roots outside the unit circle and if $e_{t}$ is iid, $E e_{t}=0$, and $E e_{t}^{2}=\sigma_{e}^{2}<\infty$, then, under $H_{0}$,

$$
D \stackrel{d}{\rightarrow} V M(s-1)
$$

Following Section 2.2 and using theorem 3 of Canova and Hansen (1995), I have the individual test statistics

$$
D_{j \pi / q}=\frac{2}{\hat{\sigma}_{e}^{2} N^{2}} \sum_{t=1}^{N} \hat{F}_{j t}^{\prime} \hat{F}_{j t}, \quad j<q,
$$

and

$$
D_{\pi}=\frac{1}{\hat{\sigma}_{e}^{2} N^{2}} \sum_{t=1}^{N} \hat{F}_{q t}^{2}, \quad j=q .
$$

The individual test statistics can be calculated as a byproduct of the joint test. Their asymptotic distribution is given in Theorem 2.

Theorem 2. Under the conditions in Theorem 1, (1) for $j<q, D_{j \pi / q} \stackrel{d}{\rightarrow} V M(2)$, and (2) $j=q, D_{\pi} \stackrel{d}{\rightarrow} V M(1)$.

The individual tests supply us with more information about the nature of the seasonal process when there is seasonal nonstationarity in the joint test. Nonstationarity can be caused by the unit roots at the individual seasonal frequencies.

The consistency of the joint and individual tests under $H_{1}$ can be obtained via the method described by Leybourne and McCabe (1994). My test is a generalization of the Leybourne and $\mathrm{McC}$ abe test at zero frequency to the seasonal frequency, whereas the $\mathrm{CH}$ test is a generalization of the KPSS test. Both tests have the same limiting distribution. If I analyze the advantages of the $D$ test, first I should begin by comparing my test with the $\mathrm{CH}$ test. The $\mathrm{CH}$ test accounts for autocorrelation in a nonparametric fashion, but in finite-samples this can cause problems if the data structure contains higher-order terms in the AR polynomial. The nonparametric adjustment then is not able to capture the serial correlation in data. My test focuses on this problem. Autocorrelation is allowed by introducing lagged terms in $y_{t}$. This parametric correction is the main advantage of the test and, with a significant AR component in the data, this results in better finite-sample performance. According to Leybourne and McCabe (1994), the test statistic is consistent at a rate $O_{p}(N)$ under $H_{1}$, but in the KPSS test this rate is $O_{p}(N / z)$ ( $z$ is the bandwidth parameter in KPSS and $\mathrm{CH}$ tests). These rates also apply to my test statistic and the $\mathrm{CH}$ test statistic under $H_{1}$. Therefore, I expect my test's power to be better.

\section{MONTE CARLO STUDY}

To examine the size and power properties of the proposed test statistics, a Monte Carlo exercise is conducted. Two quarterly models are considered. The first model is

$$
\Phi(L) y_{t}=\mu+\sum_{j=1}^{2} f_{j t}^{\prime} \gamma_{j t}+e_{t}, \quad e_{t} \sim N(0,1)
$$

and

$$
\gamma_{t}=\delta \gamma_{t-1}+u_{t}, \quad u_{t}, \sim N\left(0, \sigma_{u}^{2} G\right),
$$

where $\gamma_{0}=[1,1,1], \gamma_{t}=\left(\gamma_{1 t}, \gamma_{2 t}\right)^{\prime}$, and $0<\delta \leq 1 . \gamma_{2 t}$ is a $2 \times 1$ vector, and $G$ is a $3 \times 3$ matrix, $\sigma_{e}^{2}=1$. $\Phi(L) y_{t}$ is an 
$\operatorname{AR}(p)$ process. The second model is given by

$$
y_{t}=\mu+\sum_{j=1}^{2} f_{j t}^{\prime} \gamma_{j t}+\tau(L) e_{t}, \quad e_{t}, \sim N(0,1),
$$

and

$$
\gamma_{t}=\delta \gamma_{t-1}+u_{t}, \quad u_{t}, \sim N\left(0, \sigma_{u}^{2} G\right),
$$

where $\tau(L)=1+\tau_{1} L+\tau_{2} L^{2}+\cdots+\tau_{\ell} L^{\ell}$. The model (18)-(19) ensures a fair comparison between the $D$ test and the $\mathrm{CH}$ test because my test captures an $\operatorname{AR}(p)$ type of autocorrelation. In calculating the size of the tests, I explore the more empirically relevant case of $0<\delta<1$ and $\sigma_{u}^{2} \neq 0$. For both models, three different data-generating processes (DGP's) are used under the alternative hypothesis-

$$
\begin{aligned}
& \text { DGP1: } G=\left[\begin{array}{lll}
1 & 0 & 0 \\
0 & 0 & 0 \\
0 & 0 & 0
\end{array}\right], \\
& \text { DGP2: } G=\left[\begin{array}{lll}
0 & 0 & 0 \\
0 & 1 & 0 \\
0 & 0 & 1
\end{array}\right],
\end{aligned}
$$

and

$$
\text { DGP3: } G=\left[\begin{array}{lll}
1 & 0 & 0 \\
0 & 1 & 0 \\
0 & 0 & 1
\end{array}\right] \text {. }
$$

Under DGP 1, there is a unit root at the $\pi$ frequency as long as $\sigma_{u}^{2} \neq 0$. The $D_{\pi}$ test is designed for this specification. There is a pair of complex conjugate roots at the $\pi / 2$ frequency under DGP2 when $\sigma_{u}^{2} \neq 0$. Similarly, when $\sigma_{u}^{2}=0$ under DGP 3 there are no unit roots, but there are unit roots at all seasonal frequencies if $\sigma_{u}^{2} \neq 0$. One important fact to note is that the covariance structure implied by $G$ is different from the one that is used to construct the $D$ and $D_{\pi / 2}$ test statistics.

In the simulations, the order of the AR polynomial $p$ and the order of the MA polynomial $\ell$ are 1 and 2 . Both the AR parameters of (16)-(17) and the MA parameters of (18)-(19) are chosen carefully to understand the effect of autocorrelation on the test statistics that are proposed in this article. I set $\delta=.8$. The signal-to-noise ratio $\rho=\sigma_{u}^{2} / \sigma_{e}^{2}$ takes the value of .05 . I vary the sample size among $N=50,100,200$. I have 1,000 independent realizations for each DGP and parameter configuration. The test statistics are calculated for unit roots at all, $\pi$, and $\pi / 2$ seasonal frequencies. Robustness issues are explored in Subsection 3.3.

My test's finite-sample properties are compared to those of the $\mathrm{CH}$ tests (with and without one lag of the dependent variable included). Hylleberg (1995) showed that the $\mathrm{CH}$ test with one lag has low power. Because I analyze various data structures, however, I want to include that test in my

\begin{tabular}{|c|c|c|c|c|c|c|c|c|c|c|}
\hline \multirow[b]{2}{*}{$D G P$} & \multirow[b]{2}{*}{$S S$} & \multicolumn{3}{|c|}{$D$} & \multicolumn{3}{|c|}{$\mathrm{CH}_{0}$} & \multicolumn{3}{|c|}{$\mathrm{CH}_{1}$} \\
\hline & & $J$ & $\pi$ & $\pi / 2$ & $J$ & $\pi$ & $\pi / 2$ & $J$ & $\pi$ & $\pi / 2$ \\
\hline \multicolumn{11}{|c|}{$y_{t}+.8 y_{t-1}=\mu+f_{t}^{\prime} \gamma_{t}+e_{t}$} \\
\hline Size & 200 & 12.9 & 8.8 & 13.5 & 8.9 & 24.9 & .7 & 6.2 & 3.7 & 7.0 \\
\hline Size & 100 & 13.4 & 11.2 & 11.7 & 10.1 & 29.6 & .5 & 3.4 & 7.5 & 7.3 \\
\hline Size & 50 & 14.8 & 15.0 & 10.1 & 13.0 & 34.6 & .7 & 2.3 & 3.9 & 5.1 \\
\hline DGP3 & 200 & 99.4 & 35.3 & 89.0 & 95.8 & 69.2 & 80.1 & 91.0 & 43.3 & 95.1 \\
\hline DGP3 & 100 & 88.5 & 23.3 & 77.2 & 77.9 & 58.9 & 54.9 & 83.1 & 25.9 & 83.3 \\
\hline DGP3 & 50 & 54.6 & 30.4 & 48.5 & 53.2 & 45.0 & 45.1 & 52.8 & 17.0 & 52.6 \\
\hline DGP2 & 200 & 97.9 & 1.5 & 98.2 & 90.6 & 4.3 & 97.4 & 94.7 & 14.0 & 95.5 \\
\hline DGP2 & 100 & 84.3 & 4.7 & 86.3 & 64.4 & 6.6 & 85.9 & 79.2 & 16.0 & 80.1 \\
\hline DGP2 & 50 & 59.1 & 12.5 & 51.4 & 20.5 & 9.2 & 50.4 & 47.0 & 8.7 & 51.9 \\
\hline DGP1 & 200 & 48.0 & 56.0 & .9 & 68.1 & 71.5 & .0 & 5.3 & 12.9 & 2.7 \\
\hline DGP1 & 100 & 35.7 & 45.0 & 1.6 & 54.9 & 57.4 & .1 & 4.6 & 11.1 & 3.2 \\
\hline DGP1 & 50 & 24.8 & 31.2 & 2.4 & 40.3 & 40.8 & 8.7 & 3.9 & 12.6 & 20.0 \\
\hline \multicolumn{11}{|c|}{$y_{t}+.8 y_{t-2}=\mu+f_{t}^{\prime} \gamma_{t}+e_{t}$} \\
\hline Size & 200 & 17.3 & 16.7 & 12.0 & 40.9 & .6 & 55.5 & 49.9 & 1.8 & 63.8 \\
\hline Size & 100 & 21.0 & 14.3 & 16.6 & 47.7 & .6 & 63.7 & 50.4 & 1.0 & 64.1 \\
\hline Size & 50 & 23.6 & 10.7 & 20.4 & 44.7 & 1.5 & 61.1 & 45.7 & 1.5 & 58.8 \\
\hline DGP3 & 200 & 94.0 & 83.0 & 47.5 & 94.1 & 64.1 & 86.3 & 93.3 & 61.5 & 85.5 \\
\hline DGP3 & 100 & 81.1 & 61.9 & 41.6 & 78.9 & 42.1 & 74.3 & 77.9 & 44.2 & 73.0 \\
\hline DGP3 & 50 & 34.5 & 40.1 & 19.7 & 62.0 & 38.6 & 61.0 & 65.1 & 32.9 & 59.2 \\
\hline DGP2 & 200 & 65.1 & .4 & 72.4 & 84.9 & .1 & 85.7 & 84.8 & .0 & 85.9 \\
\hline DGP2 & 100 & 51.8 & .4 & 59.1 & 75.0 & .3 & 74.9 & 74.7 & 2.0 & 75.6 \\
\hline DGP2 & 50 & 13.7 & .8 & 14.6 & 60.4 & 8.2 & 62.2 & 59.4 & .2 & 56.8 \\
\hline DGP1 & 200 & 85.3 & 91.0 & 2.6 & 56.4 & 89.4 & 7.3 & 55.9 & 90.1 & 6.7 \\
\hline DGP1 & 100 & 66.3 & 73.1 & 10.5 & 31.7 & 67.8 & 10.7 & 30.4 & 68.9 & 16.5 \\
\hline DGP1 & 50 & 29.5 & 39.9 & 15.5 & 19.1 & 41.0 & 16.8 & 24.1 & 40.9 & 17.0 \\
\hline
\end{tabular}
study also. The underlying model of the $\mathrm{CH}$ test is the same as my model (16)-(17) and (20)-(22), but they assume $p=0$ or $p=1$. Because they also assume an $\alpha$-mixing process for

Table 1. Size and Power Comparison Between the $\mathrm{CH}$ and D tests: $A R$ Model

NOTE: In both AR parameterizations for the size part, $\gamma_{t}=.8 \gamma_{t-1}+u_{t}$. For the power part of the program, $\gamma_{t}=\gamma_{t-1}+u_{t}$. SS is the sample size. $D$, $\mathrm{CH}_{0}$, and $\mathrm{CH} \mathrm{H}_{1}$ are the $D$ and the $\mathrm{CH}$ tests with no lags and one lag, respectively. $J, \pi$, and $\pi / 2$ are the tests at all, semiannual, and annual seasonal frequencies, respectively. The DGP column shows the size and the power of the tests (DGP3, DGP2, DGP1). 
$e_{t}$, their estimates of long-run covariance matrices $\hat{\Omega}_{1}^{f}$ and $\hat{\Omega}_{0}^{f}$, for $p=1$ and $p=0$, respectively, depend on the choice of the kernel and the lag truncation number $z$. In this study the Bartlett kernel is used and, following Andrews (1991), $z=3,4,6$ is selected for $N=50,100,200$, respectively. One important point about my test is the choice of $p$, the number of lags in $y_{t}$. The $D$ tests are carried out with lag lengths chosen by the Akaike information criterion (AIC) and the Bayesian information criterion (BIC).

The results of the exercise are presented in Tables 1 and 2. The percentage of rejection of the null is given at the $5 \%$ significance level. Because the size of the $D$ and $\mathrm{CH}$ tests that are calculated in Tables 1 and 2 vary considerably, $\mathbb{I}$ calculate the size-adjusted power. In the tables, the power of the tests are size-adjusted power. The critical values for calculating these can be obtained from me on demand.

There are three Monte Carlo studies that compare the relative performance of the tests for seasonal stability. One is by Hylleberg (1995) in which the HEGY tests are contrasted with the CH tests. Ghysels, Lee, and Noh (1994) compared the performance of the HEGY test with the Dickey, Hasza, and Fuller (1984) tests. Canova and Hansen (1995) contrasted the $\mathrm{CH}$ tests with HEGY tests, but the DGP is different from the Monte Carlo study of Hylleberg (1995).

\subsection{Size and Power of the Test: $\operatorname{AR}(p)$ Process}

In this section the size and power properties of the $D$ test are compared with the $\mathrm{CH}$ test under the model (16)-(17). When analyzing the size of the tests in Table $1, \delta$ is selected to be .8 because this value corresponds to a "near" seasonal unit root. In calculating the power of the tests in Tables 1 and $2, \mathrm{I}$ set $\delta=1$.

In Table 1 , it is easy to see that the size of my tests is slightly above the nominal size of $5 \%$ in most of the cases. The $\mathrm{CH}$ tests have large size distortions for AR(2) parameterization, however. For example, for $N=200$ in an AR(2) framework, the size of the joint $D$ test is $17 \%$, whereas the joint $\mathrm{CH}$ tests reject the true null in $41-50 \%$ of the trials.

The $D$ tests have good power under different alternatives. For $N=100$, the power of the joint test is $84 \%$ when there are seasonal unit roots present at the $\pi / 2$ frequency (DGP2). For $N=200$, in an AR(2) process, the power of the joint test is $85 \%$ when there is a seasonal unit root at the $\pi$ frequency (DGP1).

The $\mathrm{CH}$ tests have mixed results under an AR structure. For $N=100$, in an AR(1) process the joint test has 64$79 \%$ power against $\mathrm{DGP} 2$. The $\mathrm{CH}$ tests with one lag of the dependent variable $\left(\mathrm{CH}_{1}\right.$ in Tables $\left.1-2\right)$ perform quite poorly in an AR(1) structure. The power is near the nominal size of the tests. Both $\mathrm{CH}$ tests also have trouble in an $\mathrm{AR}(2)$ structure when only a seasonal unit root at the $\pi$ frequency is present (DGP1). For $N=200$, the joint tests have $56 \%$ power under DGP1.

Overall, the $\mathrm{CH}$ tests do not perform well near seasonal unit roots. They suffer from size distortion. On the other hand, the proposed tests have good size and power. The $\mathrm{CH}$ tests performed well in the Monte Carlo study of Canova

Table 2. Comparison of Size and Power: The CH and D tests in an MA Model

\begin{tabular}{|c|c|c|c|c|c|c|c|c|c|c|}
\hline \multirow[b]{2}{*}{$D G P$} & \multirow[b]{2}{*}{ SS } & \multicolumn{3}{|c|}{$D$} & \multicolumn{3}{|c|}{$\mathrm{CH}_{0}$} & \multicolumn{3}{|c|}{$\mathrm{CH}_{1}$} \\
\hline & & $J$ & $\pi$ & $\pi / 2$ & $J$ & $\pi$ & $\pi / 2$ & $J$ & $\pi$ & $\pi / 2$ \\
\hline \multicolumn{11}{|c|}{$y_{t}=\mu+\sum_{j=1}^{2} f_{j t}^{\prime} \gamma_{j t}+\varepsilon_{t}+\tau \varepsilon_{t-1}$} \\
\hline Size & 200 & 9.5 & 8.8 & 6.0 & 8.0 & 9.3 & 6.6 & 8.9 & 11.6 & 7.4 \\
\hline Size & 100 & 9.8 & 9.5 & 5.4 & 4.1 & 6.4 & 5.5 & 9.0 & 14.6 & 6.8 \\
\hline Size & 50 & 8.4 & 6.9 & 7.4 & 1.7 & 4.7 & 4.4 & 4.7 & 8.7 & 6.7 \\
\hline DGP3 & 200 & 92.3 & 51.9 & 68.5 & 98.7 & 83.0 & 92.9 & 98.8 & 78.5 & 93.6 \\
\hline DGP3 & 100 & 73.4 & 52.4 & 43.7 & 91.8 & 70.3 & 75.2 & 91.6 & 67.0 & 71.1 \\
\hline DGP3 & 50 & 67.2 & 48.9 & $30: 3$ & 70.4 & 60.1 & 42.2 & 65.0 & 54.5 & 39.9 \\
\hline DGP2 & 200 & 88.1 & .0 & 93.6 & 89.1 & 0.0 & 93.9 & 86.2 & .0 & 96.2 \\
\hline DGP2 & 100 & 62.9 & .0 & 75.8 & 69.7 & 0.0 & 80.3 & 64.8 & .0 & 77.7 \\
\hline DGP2 & 50 & 24.4 & .1 & 40.5 & 34.4 & 0.0 & 48.2 & 31.5 & .0 & 45.7 \\
\hline DGP1 & 200 & 56.9 & 61.0 & 3.2 & 73.3 & 82.9 & 2.2 & 71.9 & 81.0 & 1.6 \\
\hline DGP1 & 100 & 43.3 & 53.5 & 0.6 & 65.6 & 73.5 & 1.4 & 61.5 & 70.2 & 1.4 \\
\hline DGP1 & 50 & 41.8 & 50.1 & 3.6 & 43.0 & 56.1 & 4.0 & 40.3 & 53.7 & 3.1 \\
\hline \multicolumn{11}{|c|}{$y_{t}=\mu+\sum_{j=1}^{2} f_{j t}^{\prime} \gamma_{j t}+\varepsilon_{t}+\tau \varepsilon_{t-2}$} \\
\hline Size & 200 & 14.1 & 17.4 & 9.9 & 3.0 & 9.4 & 1.1 & 1.6 & 7.2 & 1.8 \\
\hline Size & 100 & 14.4 & 18.0 & 6.7 & 2.6 & 8.6 & .6 & 1.5 & 9.3 & 1.0 \\
\hline Size & 50 & 5.6 & 17.4 & 0.7 & 1.6 & 11.3 & .1 & 1.4 & 10.8 & .2 \\
\hline DGP3 & 200 & 97.8 & 42.2 & 93.0 & 99.7 & 73.4 & 98.9 & 99.5 & 73.1 & 99.5 \\
\hline DGP3 & 100 & 92.2 & 29.8 & 90.3 & 94.2 & 55.2 & 90.7 & 93.9 & 51.9 & 94.1 \\
\hline DGP3 & 50 & 64.5 & 13.9 & 78.0 & 71.4 & 34.6 & 76.0 & 69.5 & 29.5 & 74.7 \\
\hline DGP2 & 200 & 91.1 & .8 & 93.9 & 97.2 & 2.8 & 99.3 & 95.7 & 2.7 & 98.8 \\
\hline DGP2 & 100 & 78.5 & 1.6 & 89.4 & 88.3 & 3.7 & 94.2 & 87.1 & 3.4 & 93.3 \\
\hline DGP2 & 50 & 50.3 & 1.4 & 77.4 & 55.6 & 4.3 & 75.1 & 52.6 & 4.2 & 72.2 \\
\hline DGP1 & 200 & 63.5 & 72.3 & .0 & 64.8 & 75.8 & .0 & 64.9 & 72.9 & .0 \\
\hline DGP1 & 100 & 45.4 & 54.1 & .0 & 45.9 & 54.7 & .0 & 41.8 & 53.1 & .0 \\
\hline DGP1 & 50 & 31.5 & 34.5 & .0 & 24.1 & 31.6 & .1 & 15.9 & 27.6 & .1 \\
\hline
\end{tabular}

NOTE: $\tau=.8$ in the above parameterizations. For further information on this table, see note to Table 1. 
and Hansen (1995) because of the structure of the DGP that they used. Their simulated models are not "near" seasonal unit roots at various frequencies, so it is difficult to determine the size in their study appropriately. In our case the simulated models correspond to an "almost" seasonal nonstationary case.

\subsection{Size and Power of the Test: MA(1) Process}

In this section the DGP is (18)-(19), which is the case with MA $(\ell)$ errors. This kind of setup provides a neutral ground for comparing our tests with the $\mathrm{CH}$ tests. Two types of MA processes are explored in Table 2. First I use

$$
y_{t}=\mu+\sum_{j=1}^{2} f_{j t}^{\prime} \gamma_{j t}+e_{t}+\tau e_{t-1} .
$$

Then the following MA(2) process is analyzed:

$$
y_{t}=\mu+\sum_{j=1}^{2} f_{j t}^{\prime} \gamma_{j t}+e_{t}+\tau e_{t-2} .
$$

I set $\delta=.8, \tau=.8$, and $\sigma_{u}^{2}=.05$. Using the AIC and BIC, the optimal AR lag length $p$ turned out to be 3,5, and 6 , for $N=50,100,200$, respectively.

Table 2 shows that the proposed $D$ tests have good size. The test at the $\pi / 2$ frequency performs well even in the small samples. For example, for $N=50$ in an MA(1) process, the size is $7 \%$. Even though the test at the $\pi$ frequency performs well in an MA(1) model, however, the size rises above the nominal level and is around $9-21 \%$ in an $\mathrm{MA}(2)$ setup.

The $\mathrm{CH}$ tests also have good size properties. For example, the size of the joint $\mathrm{CH}$ test with no lags of the dependent variable $\left(\mathrm{CH}_{0}\right)$ is $2-11 \%$. The sizes of both tests do not seem to be affected by the sample size.

Both the $D$ and $\mathrm{CH}$ tests have good power under different alternatives. Note, however, that the asymptotic rejection frequency of the $D$ tests is better than that of the $\mathrm{CH}$ tests. These results were given by Caner (1996).

\subsection{The Robustness Experiments}

The results are robust to overitting of the AR polynomial, correlatedness of $u_{t}$ and $e_{t}$, and overdifferencing of $y_{t}$. Specifically, when I tried fitting up to six lags for AR(1) and AR(2) models, there were no significant changes in the power and size of the test. The finite-sample properties of the test were also analyzed by using various $\sigma_{u}^{2}$ 's and $\delta$ 's. The size and the power of the test were not affected by the changes in $\sigma_{u}^{2}$. Smaller $\delta$ and AR coefficients resulted in better size properties for my test. Monte Carlo designs with longer AR polynomials such as 3 and 4 were tried, generating results that were very similar to the case of $A R(2)$ design in Table 1.

\section{CONCLUSION}

This article proposes a locally best invariant test for de- tecting the presence of seasonal unit roots in time series models. The null hypothesis of the proposed test is seasonal stationarity, whereas the seasonal unit-root hypothesis forms the alternative. The derived asymptotic distribution is nonstandard and covers serially correlated processes. My test is similar to the $\mathrm{CH}$ test for seasonal stability. The main difference between the two arises from handling autocorrelation under the respective null and alternative hypotheses. My test has a parametric correction, but the $\mathrm{CH}$ test has a nonparametric adjustment for autocorrelation. According to my simulations the $\mathrm{CH}$ test suffers from size distortion in an AR model, whereas the proposed test has good size and power. Moreover, even with different autocorrelation structures and data-generating processes, the proposed tests have good finite-sample properties.

\section{ACKNOWLEDGMENTS}

I thank Bruce Hansen for helpful advice, encouragement, and comments. I also thank Bent Sorensen for letting me use his UNIX workstation and the editor, associate editor, and two anonymous referees whose comments improved the article considerably.

\section{APPENDIX: DERIVATION OF THEOREMS}

Before proving the theorems, I need to prove a lemma and introduce some notation. Let $\Rightarrow$ denote weak convergence on $[0,1]$ with respect to the uniform metric, let $[\cdot]$ denote integer part, and let $\stackrel{p}{\rightarrow}$ denote convergence in probability.

Lemma 1.

$$
\frac{1}{\sqrt{N}} \hat{F}_{[N r]}=\frac{1}{\sqrt{N}} \sum_{t=1}^{[N r]} f_{t} \hat{e}_{t} \Rightarrow B(r)-r B(1),
$$

where $B(r)$ is a three-dimensional Brownian motion with covariance matrix $\Omega^{f}$.

Proof of Lemma 1. From the structural model under the null hypotheses, I obtain

$$
f_{t} \hat{e}_{t}=f_{t}(\mu-\hat{\mu})+f_{t} f_{t}^{\prime}\left(\gamma_{0}-\hat{\gamma}_{0}\right)
$$

$$
+f_{t} \sum_{l=1}^{p}\left(\phi_{l}-\phi_{l}^{*}\right) y_{t-l}+f_{t} e_{t}
$$

From the first-order conditions, I know that $1 / N \sum_{t=1}^{N} f_{t} \hat{e}_{t}$ $=0$, so I have

$$
\begin{aligned}
& 0=\frac{\left(\gamma_{0}-\hat{\gamma}_{0}\right)}{N} \sum_{t=1}^{N} f_{t} f_{t}^{\prime} \\
& \quad+\sum_{l=1}^{p}\left(\phi_{l}-\phi_{l}^{*}\right) \frac{1}{N} \sum_{t=1}^{N} f_{t} y_{t-l}+\frac{1}{N} \sum_{t=1}^{N} f_{t} e_{t} .
\end{aligned}
$$

Then subtract (A.2) from (A.1) to get 


$$
\begin{aligned}
f_{t} \hat{e}_{t}= & f_{t}(\mu-\hat{\mu})+\left(\gamma_{0}-\hat{\gamma}_{0}\right)\left[f_{t} f_{t}^{\prime}-\sum_{t=1}^{N} \frac{f_{t} f_{t}^{\prime}}{N}\right] \\
& +\sum_{l=1}^{p}\left(\phi_{l}-\phi_{l}^{*}\right)\left[f_{t} y_{t-l}-\sum_{t=1}^{N} \frac{f_{t} y_{t-l}}{N}\right] \\
& +\left[f_{t} e_{t}-\frac{1}{N} \sum_{t=1}^{N} f_{t} e_{t}\right] .
\end{aligned}
$$

In this expression each term will be examined in detail. Now, observe that, from the first term on the right side of (A.3),

$$
\frac{1}{\sqrt{N}} \sum_{t=1}^{[N r]} f_{t}(\mu-\hat{\mu})
$$

In (A.4), if $[N r]$ is a multiple of $s$, then (A.4) is 0 because $f_{t}$ is a zero-mean process. If $[N r]$ is not a multiple of $s$ when $N \rightarrow \infty$, (A.4) converges to 0 ,

$$
\sup _{0 \leq r \leq 1}\left|\frac{1}{\sqrt{N}} \sum_{t=1}^{[N r]} f_{t}\right| \stackrel{p}{\rightarrow} 0,
$$

and, from Potscher (1991), $(\mu-\hat{\mu})$ is $o_{p}(1)$.

The same procedure applies to the second term on the right side of (A.3) as well.

For the third term, under the null hypothesis $y_{t-l}$ is an $\operatorname{AR}(p)$ process. Following from the invariance principle for linear processes (Phillips and Solo 1992),

$$
\frac{1}{\sqrt{N}} \sum_{t=1}^{[N r]}\left(f_{t} y_{t-l}-\frac{1}{N} \sum_{t=1}^{N} f_{t} y_{t-1}\right)
$$

converges weakly and is $O_{P}(1)$. Then, from Potscher $(1991), \mathbb{I}$ know that $\left(\phi_{l}-\phi_{l}^{*}\right)$ is $o_{p}(1)$, so

$$
\begin{array}{r}
\sum_{l=1}^{p}\left(\phi_{l}-\phi_{l}^{*}\right) N^{-1 / 2} \sum_{t=1}^{[N r]}\left(f_{t} y_{t-1}-\frac{1}{N} \sum_{t=1}^{N} f_{t} y_{t-1}\right) \\
=o_{p}(1) .
\end{array}
$$

Finally, invoking the functional central limit theorem (Billingsley 1968),

$$
\begin{aligned}
& \frac{1}{\sqrt{N}} \sum_{t=1}^{[N r]}\left(f_{t} e_{t}-\frac{1}{N} \sum_{t=1}^{N} f_{t} e_{t}\right) \\
& =\frac{1}{\sqrt{N}} \sum_{t=1}^{[N r]} f_{t} e_{t}-\frac{[N r]}{N} \frac{1}{\sqrt{N}} \sum_{t=1}^{N} f_{t} e_{t} \\
& \Rightarrow B(r)-r B(1),
\end{aligned}
$$

where $B(r)$ is a vector Brownian motion with covariance matrix $\Omega^{f}$. Combining (A.5), (A.6), and (A.7), I obtain

$$
\begin{aligned}
& \frac{1}{\sqrt{N}} \sum_{t=1}^{[N r]} f_{t} \hat{e}_{t} \\
& =\frac{1}{\sqrt{N}} \sum_{t=1}^{[N r]}\left(f_{t} e_{t}-\frac{1}{N} \sum_{t=1}^{N} f_{t} e_{t}\right)
\end{aligned}
$$

$$
\begin{aligned}
& +\frac{1}{\sqrt{N}} \sum_{t=1}^{[N r]} \sum_{l=1}^{p}\left(\phi_{l}-\phi_{l}^{*}\right)\left[f_{t} y_{t-1}-\sum_{t=1}^{N} \frac{f_{t} y_{t-l}}{N}\right] \\
& +\frac{1}{\sqrt{N}} \sum_{t=1}^{[N r]}\left(\gamma_{0}-\hat{\gamma}_{0}\right)\left[f_{t} f_{t}^{\prime}-\sum_{t=1}^{N} \frac{f_{t} f_{t}^{\prime}}{N}\right] \\
& +(\mu-\hat{\mu}) \sum_{t=1}^{N} \frac{f_{t}}{\sqrt{N}} \\
& \Rightarrow B(r)-r B(1) .
\end{aligned}
$$

Proof of Theorem 1. From Lemma 1 and applying the continuous mapping theorem, I obtain

$$
\begin{aligned}
D & =\frac{1}{N^{2}} \sum_{t=1}^{N} \hat{F}_{t}^{\prime}\left(\hat{\Omega}^{f}\right)^{-1} \hat{F}_{t} \\
& \Rightarrow \int_{0}^{1} W_{s-1}(r)^{\prime} W_{s-1}(r) d r \\
& =V M(s-1) .
\end{aligned}
$$

Proof of Theorem 2. This theorem is proved in a manner similar to the proof of Theorem 1.

[Received September 1996. Revised May 1997.]

\section{REFERENCES}

Andrews, D. (1991), "Heteroskedasticity and Autocorrelation Consistent Covariance Matrix Estimation,” Econometrica, 59, 817-858.

Ansley, C. F., and Newbold, P. (1980), "Finite Sample Properties of Estimators for Autoregressive Moving Average Models," Journal of Econometrics, 13, 159-183.

Billingsley, P. (1968), Convergence of Probability Measures, New York: Wiley.

Caner, M. (1996), “A Locally Optimal Seasonal Unit Root Test," working paper, Koc University, Dept. of Economics.

Canova, F., and Hansen, B. E. (1995), "Are Seasonal Patterns Constant Over Time? A Test for Seasonal Stability," Journal of Business \& Economic Statistics, 13, 237-252.

Dickey, D., and Fuller, W. (1979), "Distribution of the Estimators for Autoregressive Time Series With a Unit Root," Journal of the American Statistical Association, 84, 427-431.

Dickey, D., Hasza, D., and Fuller, W. (1984), "Testing for Unit Roots in Seasonal Time Series," Journal of the American Statistical Association, 79, 355-367.

Galbraith, J. W., and Zinde-Walsh, V. (1994), "A Simple Noniterative Estimator for Moving Average Models," Biometrika, 81, 143-155.

Ghysels, E., Lee, H., and Noh, J. (1994), "Testing for Unit Roots in Seasonal Time Series: Some Theoretical Extension and a Monte Carlo Investigation," Journal of Econometrics, 62, 415-442.

Hannan, E. J. (1970), Multiple Time Series, New York: Wiley.

Hansen, B. E. (1990), "Lagrange Multipler Tests for Parameter Instability in Nonlinear Models," working paper, University of Rochester, Dept. of Economics.

(1992), "Testing for Parameter Instability in Linear Models," Journal of Policy Modeling, 14, 517-533.

Harvey, A. C. (1989), Forecasting, Structural Time Series Models and the Kalman Filter, Cambridge, U.K.: Cambridge University Press.

Hylleberg, S. (1995), "Tests for Seasonal Unit Roots: A Comparative Study," Journal of Econometrics, 69, 5-25.

Hylleberg, S., Engle, R., Granger, C., and Yoo, S. (1990), "Seasonal Integration and Cointegration," Journal of Econometrics, 44, 215-238. 
King, M. L., and Hillier, G. H. (1985), "Locally Best Invariant Tests of the Error Covariance Matrix of the Linear Regression Model," Journal of the Royal Statistical Society, Ser. B, 47, 98-102.

Kwiatkowski, D., Phillips, P. C. B., Schmidt, P., and Shin, Y. (1992), “Testing the Null Hypothesis of Stationarity Against the Alternative of a Unit Root: How Sure Are We That Economic Time Series Have a Unit Root?" Journal of Econometrics, 44, 159-178.

Leybourne, S. J., and McCabe, B. P. M. (1994), “A Consistent Test for A Unit Root," Journal of Business \& Economic Statistics, 12, 157-167.

Nyblom, J. (1989), "Testing for the Constancy of Parameters Over Time," Journal of the American Statistical Association, 84, 223-230.
Phillips, P. C. B., and Solo, V. (1992), "Asymptotics for Linear Processes," The Annals of Statistics, 20, 971-1001.

Potscher, B. M. (1991), "Non-invertibility and Pseudo-Maximum Likelihood Estimation of Mis-specified ARMA models," Econometric Theory, 7, 435-449.

Saikkonen, P., and Luukkonen, R. (1993), "Testing for a Moving Average Unit Root in Autoregressive Integrated Moving Average Models," Journal of the American Statistical Association, 88, 596-601.

Tam, W., and Reinsel, G. (1995), "Tests for Seasonal Moving Average Unit Root in ARIMA Models," working paper, University of Wisconsin, Madison, Dept. of Statistics. 